\title{
Don’t Hold Your Breath
}

\author{
Vishal Goyal, $M D^{1,2}$ and Malathi Srinivasan, $M D^{1,3}$ \\ 'Department of Medicine, University of California, Davis, School of Medicine, Davis, CA, USA; ${ }^{2}$ Department of Internal Medicine, University of \\ New Mexico, Albuquerque, NM, USA; ${ }^{3}$ Department of Internal Medicine, UC Davis School of Medicine, Davis, CA, USA.
}

KEY WORDS: cribriform; ethmoid sinus fractures; meningocele; normal intracranial pressures; Valsalva maneuvers.

J Gen Intern Med 26(3):345

DOI: $10.1007 / \mathrm{s} 11606-010-1565-9$

(C) The Author(s) 2010. This article is published with open access at Springerlink.com

A 50-year-old athletic man presented with two weeks of debilitating "sinus" headache, continuous left nostril drainage, persistent cough, with no history of head trauma.

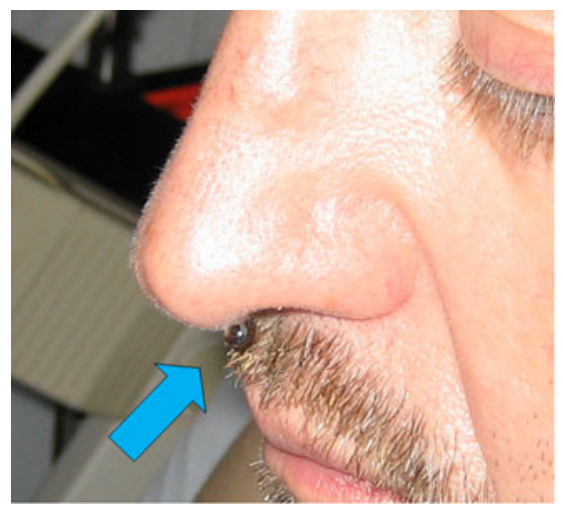

Figure 1. His nasal discharge was unilateral, worse with coughing or leaning forward.

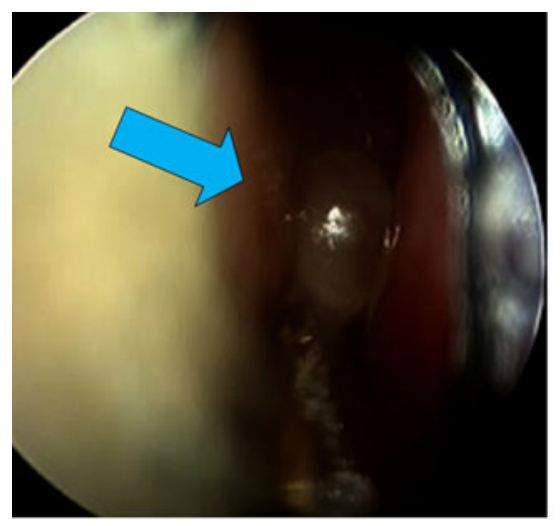

Figure 2. Endoscopy revealed a small bony defect in his left nasal cribriform plate.

Received June 5, 2010

Revised October 1, 2010

Accepted October 7, 2010

Published online November 20, 2010
His nasal discharge was unilateral, worse with coughing or leaning forward (Fig. 1), with one clear drop every 2-3 seconds. Analysis demonstrated beta-2 transferrin, a transferrin isoform found almost exclusively in cerebrospinal fluid (CSF). Brain MRI showed no cribriform or ethmoid sinus fractures. Endoscopy revealed a small bony defect in his left nasal cribriform plate (Fig. 2), with active CSF drainage, causing his rhinorrhea. After endoscopic meningocele repair, his symptoms completely resolved. Further review revealed that our patient was a former US Marine who exercised aggressively, bench-pressing 200-300lbs daily, but with poor technique-holding his breath while lifting. Normal intracranial pressures (ICPs) range from $5-15 \mathrm{cmH}_{2} \mathrm{O}$ when laterally recumbent ${ }^{1}$. Patients with CSF leaks, regardless of etiology, have ICPs of $26-33 \mathrm{cmH}_{2} \mathrm{O}$, suggesting correlations between intracranial hypertension and CSF leak ${ }^{2}$. When fifteen patients were placed in left lateral recumbent positions and asked to bear-down against a closed glottis (Valsalva), every patient achieved ICPs of $25 \mathrm{cmH}_{2} \mathrm{O}$ or greater, with one reaching $47 \mathrm{cmH}_{2} \mathrm{O}^{3}$. People performing frequent Valsalva maneuvers, including weightlifting without exhaling, elevate their ICPs to pressures associated with spontaneous CSF leaks. Smaller leaks may spontaneously heal. Large, symptomatic or continuous leaks should be repaired to prevent meningitis. Large or rapidly draining defects may be visualized during endoscopy. Smaller defects are localized with dyes injected into CSF. We presume that our patient's Valsalva maneuvers during weightlifting elevated his ICP, eroded his meninges, eventually causing an meningocele and spontaneous CSF rhinorrhea.

Conflict of interest: None disclosed.

Open Access: This article is distributed under the terms of the Creative Commons Attribution Noncommercial License which permits any noncommercial use, distribution, and reproduction in any medium, provided the original author(s) and source are credited.

Corresponding Author: Malathi Srinivasan, MD; Department of Internal Medicine, UC Davis School of Medicine, Davis, CA, USA (e-mail:malathi@ucdavis.edu).

\section{REFERENCES}

1. Wise SK, Schlosser RJ. Evaluation of spontaneous nasal cerebrospinal fluid leaks. Current Opinion in Otolaryngology \& Head and Neck Surgery. 2007;15(1):28-34.

2. Schlosser RJ, Wilensky EM, Grady MS, Bolger WE. Elevated intracranial pressures in spontaneous cerebrospinal fluid leaks. American Journal of Rhinology. 2003;17(4):191-5.

3. Neville L, Egan RA. Frequency and amplitude of elevation of cerebrospinal fluid resting pressure by the Valsalva maneuver. Canadian Journal of Ophthalmology. 2005;40(6):775-7. 